ESAIM: M2AN 48 (2014) 285-304

DOI: $10.1051 / \mathrm{m} 2 \mathrm{an} / 2013102$
ESAIM: Mathematical Modelling and Numerical Analysis

www.esaim-m2an.org

\title{
STABILIZATION OF A NON STANDARD FETI-DP MORTAR METHOD FOR THE STOKES PROBLEM
}

\author{
E. Chacón Vera ${ }^{1}$ and T. Chacón Rebollo ${ }^{2}$
}

\begin{abstract}
In a recent paper [E. Chacón Vera and D. Franco Coronil, J. Numer. Math. 20 (2012) 161-182.] a non standard mortar method for incompressible Stokes problem was introduced where the use of the trace spaces $H^{1 / 2}$ and $H_{00}^{1 / 2}$ and a direct computation of the pairing of the trace spaces with their duals are the main ingredients. The importance of the reduction of the number of degrees of freedom leads naturally to consider the stabilized version and this is the results we present in this work. We prove that the standard Brezzi-Pitkaranta stabilization technique is available and that it works well with this mortar method. Finally, we present some numerical tests to illustrate this behaviour.
\end{abstract}

Mathematics Subject Classification. 65N30, 65N55.

Received July 26, 2012. Revised June 13, 2013.

Published online January 10, 2014.

\section{INTRODUCTION}

The starting point in many domain decomposition methods is to split the computational domain $\Omega\left(\Omega \subset \mathbb{R}^{2}\right.$ bounded polygonal domain to ease presentation) into non overlapping (open) polygonal subdomains $\left\{\Omega^{s}\right\}_{s}$ with the purpose of working locally and recover a global solution after some iteration process.

Following Girault and Raviart [10], Grisvard [11] or Adams [1] we introduce some well known notation to ease the presentation: Denote by $H^{r}(\Omega)$ the usual Sobolev space endowed with the Sobolev norm $\|\cdot\|_{r, \Omega}$ and by $H_{0}^{1}(\Omega)$ the closure in $H^{1}(\Omega)$ of all the smooth functions with support inside $\Omega$. Then, we split $\Omega$, the computational domain, as follows

$$
\Omega=\cup_{s=1}^{S} \Omega^{s}, \quad \Omega^{s} \cap \Omega^{t}=\emptyset \quad \text { for } \quad s \neq t .
$$

Next, denote by $\partial \Omega^{s}$ the boundary of any of the (open) polygonal subdomains, suppose that

$$
\Gamma_{s, t}=\partial \Omega^{s} \cap \partial \Omega^{t}
$$

Keywords and phrases. Incompressible Stokes problem, non-standard FETI-DP mortar method.

1 Dpto. Matemáticas, Facultad de Matemáticas, Universidad de Murcia, Campus Espinardo, 30100 Murcia, Spain. eliseo@um.es

2 Dpto. Ecuaciones Diferenciales y Análisis Numérico, Facultad de Matemáticas, Universidad de Sevilla, Tarfia sn. 41012 Sevilla,

Spain. chacon@us.es 
is either an edge (i.e., a segment), a crosspoint or empty and, finally, consider

$$
\mathcal{E}_{0}=\left\{\Gamma_{e}\right\}_{e=1, \ldots, E}
$$

the sorted set of all edges inside $\Omega$, also known as the skeleton of the decomposition.

A common idea to devise a parallel computation of a function $u \in H_{0}^{1}(\Omega)$ is to use a variational formulation where the restriction to subdomains of the original equation and some interface terms show up. Usually, this process is performed via integration by parts and boundary terms appear as dualities. How to handle this dualities is the core of the following arguments.

A standard space for mortar methods is the product Hilbert space

$$
X_{\delta}=\left\{v \in L^{2}(\Omega) ; v^{s}=v_{\Omega_{\Omega^{s}}} \in H^{1}\left(\Omega^{s}\right) \cap H_{0}^{1}(\Omega), 1 \leq s \leq S\right\}
$$

endowed with the natural norm

$$
\|v\|_{1, \delta}=\left\{\sum_{s=1}^{S}\left\|v^{s}\right\|_{1, \Omega^{s}}^{2}\right\}^{1 / 2} .
$$

However, this space $X_{\delta}$ has the disadvantage that it does not control the jumps $[v]_{\Gamma_{e}}$ across interfaces $\Gamma_{e}$; the internal crosspoints (final points of internal edges) are the trouble. As a consequence, it seems that the following strict subspace of $X_{\delta}$

$$
X=\left\{v \in X_{\delta},[v]_{\Gamma_{e}} \in H_{00}^{1 / 2}\left(\Gamma_{e}\right), \forall \Gamma_{e} \in \mathcal{E}_{0}\right\}
$$

gives a better framework because it considers only the functions whose jumps belong to the trace spaces $H_{00}^{1 / 2}\left(\Gamma_{e}\right)$, which are the correct ones for neighboring subdomains. Endowed with the graph norm

$$
\|v\|_{X}=\left\{\sum_{s=1}^{S}\left\|v^{s}\right\|_{1, \Omega^{s}}^{2}+\sum_{e=1}^{E}\left\|[v]_{\Gamma_{e}}\right\|_{1 / 2,00, \Gamma_{e}}^{2}\right\}^{1 / 2}
$$

$X$ is a Hilbert space. Here, $\|\cdot\|_{1 / 2,00, \Gamma_{e}}$ is the norm induced by the scalar product $(\cdot, \cdot)_{1 / 2,00, \Gamma_{e}}$ on $H_{00}^{1 / 2}\left(\Gamma_{e}\right)$ given by

$$
(w, v)_{1 / 2,00, \Gamma_{e}}=(w, v)_{1 / 2, \Gamma_{e}}+\int_{\Gamma_{e}} \frac{w(x) v(x)}{d\left(x, \partial \Gamma_{e}\right)} \mathrm{d} x
$$

where $d\left(x, \partial \Gamma_{e}\right)$ is the distance of $x$ to the boundary of $\Gamma_{e}$ and

$$
(w, v)_{1 / 2, \Gamma_{e}}=\int_{\Gamma_{e}} w(x) v(x) \mathrm{d} x+\int_{\Gamma_{e}} \int_{\Gamma_{e}} \frac{(w(x)-w(y))(v(x)-v(y))}{|x-y|^{2}} \mathrm{~d} x \mathrm{~d} y .
$$

To simplify notation, we write

$$
\{w, v\}_{\Gamma_{e}}=(w, v)_{1 / 2,00, \Gamma_{e}}, \quad \forall w, v \in H_{00}^{1 / 2}\left(\Gamma_{e}\right) .
$$

Clearly, $X$ is not a Hilbert space with respect to $\|\cdot\|_{1, \delta}$ because the norm in $H_{00}^{1 / 2}\left(\Gamma_{e}\right)$ is strictly stronger than that on $H^{1 / 2}\left(\Gamma_{e}\right)$. Moreover,

$$
H_{0}^{1}(\Omega) \varsubsetneqq X \varsubsetneqq X_{\delta} .
$$


Remark 1.1. Poincare's inequality and the control we have on the jumps, interconnecting all subdomains, allow the use on $X$ of the equivalent norm $|v|_{X}^{2}=(v, v)_{X}$, where $(\cdot, \cdot)_{X}$ is the scalar product given for any $u, v \in X$ by

$$
(u, v)_{X}=\sum_{s=1}^{S}\left(\nabla u^{s}, \nabla v^{s}\right)_{\Omega^{s}}+\sum_{e=1}^{E}\left\{[u]_{\Gamma_{e}},[v]_{\Gamma_{e}}\right\}_{\Gamma_{e}},
$$

what is to say, $|v|_{X}^{2}=0$ implies $v=0$.

As far as we know, the use of the space $X_{\delta}$ was introduced by Raviart and Thomas [13] and $X$ was considered by Braess, Dahmen and Wieners [2] and by Ben Belgacem [3]. Following their analysis, one sees that $H_{0}^{1}(\Omega)$ can be identify as a subspace of $X$ and, also, as a subspace of $X_{\delta}$ by means of appropriate linear restrictions:

- In the case of $X_{\delta}$, just one linear restriction on $X_{\delta}$ that acts globally on the skeleton $\mathcal{E}_{0}$ is enough. The global action guarantees that the local functions are correctly glued together, see the characterization $(2.12)$ of $H_{0}^{1}(\Omega)$ in Raviart and Thomas [13].

- On the other hand, for $X$ we need as many linear restrictions as interfaces. But, as the coupling is already present in $X$ by definition, all of these linear restrictions are independent from each other and their local action is just to guarantee the nullity of the jump across each interface.

In both cases, these linear restrictions are identified with Lagrange multipliers. The most important issue here is the nature of these multipliers:

- in the case of $X_{\delta}$, they are a properly chosen sum of the duality pairings $H^{-1 / 2}\left(\partial \Omega^{s}\right)-H^{1 / 2}\left(\partial \Omega^{s}\right)$.

- while in the case of $X$, they are the pairing $H_{00}^{-1 / 2}\left(\Gamma_{e}\right)-H_{00}^{1 / 2}\left(\Gamma_{e}\right)$ for each of the local Lagrange multipliers.

Many popular methods for solving elliptic problems are based on the idea of forcing the nullity of the jumps across interfaces in some way. For instance, mortar methods do it in a weak sense while FETI methods impose pointwise continuity at some interface nodes. A proper formulation of the continuous problem will guarantee the stability of the discrete version. Consequently, it is interesting to discuss some of the characteristics of these two above decompositions:

First, the obtention of the jumps at the interfaces from the first approach is not possible at the continuous level. That is because at corner points the normal vector is not defined and it is not possible to split locally the action of the normal derivative, considered as a linear form, see Grisvard [11]. Therefore, the second approach seems to be safer because jumps naturally show up. Moreover, the control of the jumps only in terms of the gradient norms is impossible due to internal crosspoints. Again, the use of $X$ seems to be more interesting.

Secondly, the Lagrange multipliers are usually implemented by means of the scalar $L^{2}$ product on interfaces and end up representing the normal fluxes across these interfaces, usually some physical quantity of interest. But this procedure makes sense only when these normal fluxes are smooth enough to be identified with $L^{2}$ functions. Even when the true solution is smooth enough to guarantee smooth fluxes (something that usually comes from the regularity of the data), the norms involved in the existence analysis are usually weaker and do not see this extra regularity for the true solution.

As a consequence, we are faced with stability issues that are delicate to solve. In fact, the continuous version of the Lagrange multiplier action on each interface $\Gamma_{e}$ is the duality $H_{00}^{-1 / 2}\left(\Gamma_{e}\right)-H_{00}^{1 / 2}\left(\Gamma_{e}\right)$ which means that the regularity expected for the Lagrange multiplier is $H_{00}^{-1 / 2}$. As a consequence, assuming an $L^{2}$ regularity for this multiplier and writing this duality in terms of the scalar product in $L^{2}\left(\Gamma_{e}\right)$ requires stabilization techniques.

Although the analysis in the case of elliptic problems has been done on a discrete level using mesh dependent norms, see for instance the works by Ben Belgacem and Maday [3] or Bernardy, Maday and Patera [5], a continuous study face the question of controlling all the jumps and the difficulty of handling the duality pairing for discrete functions without wasting information.

In the previous works by Bernardi et al. $[4,5]$ and Chacón Vera et al. [6], these questions are handled by using the space $X$ and the (Riesz) identification of the Hilbert space $H_{00}^{-1 / 2}\left(\Gamma_{e}\right)$ with its dual $H_{00}^{1 / 2}\left(\Gamma_{e}\right)$ : the 
duality $H_{00}^{-1 / 2}\left(\Gamma_{e}\right)-H_{00}^{1 / 2}\left(\Gamma_{e}\right)$ is represented in terms of the scalar product in $H_{00}^{1 / 2}\left(\Gamma_{e}\right)$. As a consequence, the duality is computable and the regularity of the Lagrange multiplier now is $H_{00}^{1 / 2}$ instead of $H_{00}^{-1 / 2}$. The stability questions are solved but the disadvantage is that the Lagrange multiplier loses its physical meaning, although the normal fluxes can still be recovered from the computed solution. A similar effort can be found in the paper by Lee and Park [12].

According to these ideas, in $[4,6]$ a continuous framework is proposed for elliptic equations in terms of a saddle point problem that resembles the standard primal hybrid formulation and, sort of, falls into the FETIDP mortar familly of methods: FETI-DP alike because only continuity at cross points is imposed and mortar alike because nonmatching meshes at interfaces are allowed, although we use a different scalar product for the mortaring process. In this method, all of the previously mentioned technical difficulties are avoided and the continuous analysis holds for internal approximations even in the case of non conforming meshes, i.e., we obtain mesh independent inf-sup and continuity conditions at the discretization level. Another remarkable property is that the stability of the discretization is independent of the varying mesh sizes, i.e., it does not matter which side is the mortar.

In [7] this effort is extended to the case of the incompressible Stokes equations with mixed finite elements showing that the same results hold. In this work we study the use of stabilization techniques to make the computation less expensive. Our analysis will be presented in the two dimensional setting to simplify the presentation. These main ideas extend easily to the three dimensional situation although a detailed study is required. Finally some numerical tests are shown as a conclusion.

\section{INCOMPRESSIBLE STOKES EQUATIONS}

The splitting of the computational subdomain for the pressure is not easy because the zero average imposes a global restriction. To fix this difficulty, we introduce a new variable in the Stokes equations that sets free the pressure space from this restriction.

Incompressible Stokes equations with homogeneous boundary conditions amount to find $u \in \mathbf{H}_{0}^{1}(\Omega)=$ $\left(H_{0}^{1}(\Omega)\right)^{2}$ and $p \in L^{2}(\Omega)$ such that

$$
\begin{array}{rlrl}
(\nabla u, \nabla v)_{\Omega}-(p, \operatorname{div}(v))_{\Omega} & =(f, v)_{\Omega}, \quad \forall v \in \mathbf{H}_{0}^{1}(\Omega) \\
-(q, \operatorname{div}(u))_{\Omega} & =0, \quad \forall q \in L^{2}(\Omega) \\
\int_{\Omega} p & =0 . & &
\end{array}
$$

We add a new scalar unknown that takes the role of the pressure average as follows: we look for a pair of values $(u, \tau) \in \mathbf{H}_{0}^{1}(\Omega) \times \mathbb{R}$ and $p \in L^{2}(\Omega)$ such that

$$
\begin{array}{cc}
(\nabla u, \nabla v)_{\Omega}-(p, \operatorname{div}(v))_{\Omega}+t\left(\tau-\int_{\Omega} p\right)=(f, v)_{\Omega}, & \forall(v, t) \in \mathbf{H}_{0}^{1}(\Omega) \times \mathbb{R} \\
-(q, \operatorname{div}(u))_{\Omega}-\tau \int_{\Omega} q=0, & \forall q \in L^{2}(\Omega) .
\end{array}
$$

Evidently, $q \equiv 1$ implies $\tau=0$ and $v \equiv 0$ implies $\int_{\Omega} p=0$. This formulation can be seen as a saddle point problem if we pair the $u$ and $\tau$ variables. Set $W=\mathbf{H}_{0}^{1}(\Omega) \times \mathbb{R}$ and let $\underline{v}=(v, t) \in W$ any element of $W$. We norm $W$ by using

$$
\|\underline{v}\|_{W}^{2}=\|(v, t)\|_{W}^{2}=\|\nabla v\|_{0, \Omega}^{2}+t^{2}
$$

and let $(\cdot, \cdot)_{W}: W \times W \mapsto \mathbb{R}$ be the scalar product on $W$, i.e.,

$$
(\underline{u}, \underline{v})_{W}=((u, \tau),(v, t))_{W}=(\nabla u, \nabla v)_{\Omega}+\tau t .
$$


Take $b: W \times L^{2}(\Omega) \mapsto \mathbb{R}$ given by

$$
b(q,(v, t))=-(q, \operatorname{div}(v))_{\Omega}-t \int_{\Omega} q .
$$

Then, we look for $\underline{u}=(u, \tau) \in W$ and $p \in L^{2}(\Omega)$ such that for all $\underline{v}=(v, t) \in W$ and $q \in L^{2}(\Omega)$

$$
\begin{aligned}
(\underline{u}, \underline{v})_{W}+b(p, \underline{v}) & =(f, v)_{\Omega}, \\
b(q, \underline{u}) & =0 .
\end{aligned}
$$

While the ellipticity for this saddle point problem is trivial, we need an inf-sup condition for $b$. The following result is claimed but not fully proved in [7], we give it now:

Lemma 2.1. For any $p \in L^{2}(\Omega)$ there exists a positive constant $\beta>0$ with

$$
\sup _{(v, t) \in W} \frac{b(p,(v, t))}{\|(v, t)\|_{W}} \geq \sup _{v \in \mathbf{H}_{0}^{1}(\Omega), t \in \mathbb{R}} \frac{b(p,(v, t))}{\left(\|\nabla v\|_{0, \Omega}^{2}+t^{2}\right)^{1 / 2}} \geq \beta\|p\|_{0, \Omega} .
$$

As a consequence, problem (2.1)-(2.2) is well posed and its solution is that of the original Stokes problem with Dirichlet homogeneous boundary conditions.

Proof. Given $p \in L^{2}(\Omega)$, for any $(v, t) \in W$ we have

$$
\begin{aligned}
b(p,(v, t)) & =-(p, \operatorname{div}(v))_{\Omega}-t \int_{\Omega} p \\
& =-\left(p-p_{\Omega}, \operatorname{div}(v)\right)_{\Omega}-t|\Omega| p_{\Omega}
\end{aligned}
$$

where we use that $\int_{\Omega} \operatorname{div}(v) \mathrm{d} x=0$ and take

$$
p_{\Omega}=\frac{1}{|\Omega|} \int_{\Omega} p .
$$

Now we choose $(v, t)$ properly: as $p-p_{\Omega} \in L_{0}^{2}(\Omega)$ there exists a function $v^{\star} \in \mathbf{H}_{0}^{1}(\Omega)$ and a constant $C$ such that

$$
-\left(p-p_{\Omega}\right)=\operatorname{div}\left(v^{\star}\right), \quad\left\|\nabla v^{\star}\right\|_{0, \Omega} \leq C\left\|p-p_{\Omega}\right\|_{0, \Omega} .
$$

Then

$$
\begin{aligned}
b\left(p,\left(v^{\star}, t\right)\right) & =-\left(p-p_{\Omega}, \operatorname{div}\left(v^{\star}\right)\right)_{\Omega}-t|\Omega| p_{\Omega} \\
& =\left\|p-p_{\Omega}\right\|_{0, \Omega}^{2}-t|\Omega| p_{\Omega} .
\end{aligned}
$$

On the other hand,

$$
\left\|p-p_{\Omega}\right\|_{0, \Omega}^{2}=\|p\|_{0, \Omega}^{2}+\left\|p_{\Omega}\right\|_{0, \Omega}^{2}-2\left(p, p_{\Omega}\right)_{0, \Omega}=\|p\|_{0, \Omega}^{2}-|\Omega| p_{\Omega}^{2} .
$$

Then

$$
b\left(p,\left(v^{\star}, t\right)\right)=\|p\|_{0, \Omega}^{2}-|\Omega| p_{\Omega}^{2}-t|\Omega| p_{\Omega} .
$$

and the choice $t^{\star}=-p_{\Omega}$ gives

$$
b\left(p,\left(v^{\star}, t^{\star}\right)\right)=\|p\|_{0, \Omega}^{2} .
$$


Finally, we see that $\left\|\left(v^{\star}, t^{\star}\right)\right\|_{W} \leq C\|p\|_{0, \Omega}$. We use that

$$
\begin{gathered}
\|p\|_{0, \Omega}^{2}=\left\|p-p_{\Omega}\right\|_{0, \Omega}^{2}+|\Omega| p_{\Omega}^{2} . \\
\left\|\left(v^{\star}, t^{\star}\right)\right\|_{W}^{2}=\left\|\nabla v^{\star}\right\|_{0, \Omega}^{2}+\left(t^{\star}\right)^{2} \leq C\left\|p-p_{\Omega}\right\|_{0, \Omega}^{2}+p_{\Omega}^{2} \\
\leq \max \{C, 1\}\left\{\left\|p-p_{\Omega}\right\|_{0, \Omega}^{2}+p_{\Omega}^{2}\right\} .
\end{gathered}
$$

Then, straightforward bounds give the existence of a positive constant $\beta>0$ that only depends on the domain $\Omega$ such that

$$
\left\|\left(v^{\star}, t^{\star}\right)\right\|_{W} \leq \beta^{-1}\|p\|_{0, \Omega}
$$

and the inf-sup condition (2.3) follows with this constant $\beta>0$

$$
b\left(p,\left(v^{\star}, t^{\star}\right)\right)=\|p\|_{0, \Omega}^{2} \geq \beta\|p\|_{0, \Omega}\left\|\left(v^{\star}, t^{\star}\right)\right\|_{W} .
$$

Remark 2.2. The solution of the standard Stokes equations is associated to the saddle point $(u, p) \in$ $\left(H_{0}^{1}(\Omega)\right)^{2} \times L_{0}^{2}(\Omega)$ of the Lagrangean functional over $\left(H_{0}^{1}(\Omega)\right)^{2} \times L_{0}^{2}(\Omega)$ given by

$$
\mathcal{L}(v, q)=\frac{1}{2} \int_{\Omega}\|\nabla v\|_{0, \Omega}^{2}-\int_{\Omega} f v-\int_{\Omega} q \operatorname{div}(v)
$$

or, equivalently, with the constrained minimization of the energy problem

$$
\begin{aligned}
& \min _{v \in\left(H_{0}^{1}(\Omega)\right)^{2}}\left\{\frac{1}{2} \int_{\Omega}\|\nabla v\|_{0, \Omega}^{2}-\int_{\Omega} f v\right\} \\
& \text { subject to } \operatorname{div}(v)=0 .
\end{aligned}
$$

We have taken $V=\left(H_{0}^{1}(\Omega)\right)^{2} \times \mathbb{R}$ and considered the saddle point $((u, \tau), p) \in V \times L^{2}(\Omega)$ of the Lagrangean functional over $V \times L^{2}(\Omega)$ given by

$$
\mathcal{L}((v, t), q)=\frac{1}{2} \int_{\Omega}\|\nabla v\|_{0, \Omega}^{2}+\frac{1}{2} t^{2}-\int_{\Omega} f v-\int_{\Omega} q \operatorname{div}(v)-t \int_{\Omega} q,
$$

or, equivalently, with the constrained minimization of the new energy problem

$$
\begin{aligned}
& \min _{v \in\left(H_{0}^{1}(\Omega)\right)^{2}}\left\{\frac{1}{2} t^{2}+\frac{1}{2} \int_{\Omega}\|\nabla v\|_{0, \Omega}^{2}-\int_{\Omega} f v\right\} \\
& \text { subject to } \operatorname{div}(v)+t=0 .
\end{aligned}
$$

As a consequence, we have relaxed the energy functional in a simple way and this small change removes the zero average restriction on the pressure.

\subsection{Multisubdomain formulation}

Next we split $\Omega=\cup_{s=1}^{S} \Omega^{s}$ as in (1.1)-(1.2) such that each $\Omega^{s}$ is of area $\mathcal{O}\left(H^{2}\right)$ and shape regular while each $\Gamma_{e}$ is of length $\mathcal{O}(H)$ for some fixed $H>0$, see Figure 1 for example. This means that there exists positive constants $c_{1}, c_{2}$ and $H>0$ such that for any $e=1, \ldots, E$ and $s=1, \ldots, S$ we have

$$
c_{1} H \leq\left|\Gamma_{e}\right| \leq c_{2} H, \quad c_{1} H^{2} \leq\left|\Omega^{s}\right| \leq c_{2} H^{2},
$$

where $|\cdot|$ is the corresponding Lebesgue measure. We also assume that

$$
\mathcal{E}_{0}=\left\{\Gamma_{e}\right\}_{e=1, \ldots, E}
$$




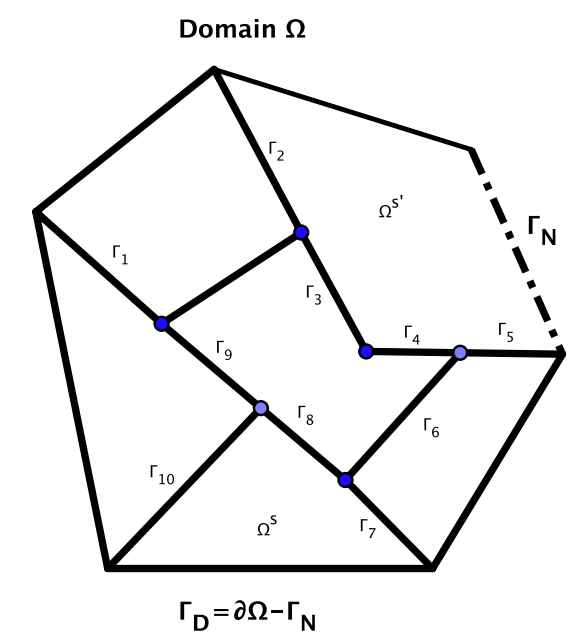

FiguRE 1. Example of domain decomposition where cross points are marked with big dots and the skeleton is the set of all the $\Gamma_{i}$ for $i=1,2,3, \ldots, 10$. In the case of Dirichlet Boundary conditions we have $\Gamma_{N}=\emptyset$.

contains the sorted set of all edges inside $\Omega$. This is now the skeleton of the decomposition. We let $\mathcal{C}$ be the set of all vertices of the polygonal subdomains $\Omega^{s}$ that are not on $\partial \Omega$; these will be called cross points. On the other hand, any open line segment, without corners or junctions with other lines, on the boundary of a subdomain between two consecutive crosspoints will be referred to as an edge, or interface, see Figure 1. Finally, we denote by $[v]_{\Gamma_{e}}$ the jump across any interface $\Gamma_{e}$. Once all the interfaces and subdomains are sorted these jumps are always computed following the same pattern.

We propose to relax jumps across interfaces and add the correct multipliers for each of these jumps. First we define our decomposition for the velocities. We take

$$
X_{\delta}=\left\{v \in L^{2}(\Omega) ; v^{s}=v_{\Omega^{s}} \in H^{1}\left(\Omega^{s}\right) \cap H_{0}^{1}(\Omega), 1 \leq s \leq S\right\},
$$

and consider $X$ given by

$$
X=\left\{v \in X_{\delta},[v]_{\Gamma_{e}} \in H_{00}^{1 / 2}\left(\Gamma_{e}\right), \forall \Gamma_{e} \in \mathcal{E}_{0}\right\}
$$

endowed with the norm $|v|_{X}^{2}=(v, v)_{X}$, where $(\cdot, \cdot)_{X}$ is the scalar product given for any $u, v \in X$ by

$$
(u, v)_{X}=\sum_{s=1}^{S}\left(\nabla u^{s}, \nabla v^{s}\right)_{\Omega^{s}}+\sum_{e=1}^{E}\left\{[u]_{\Gamma_{e}},[v]_{\Gamma_{e}}\right\}_{\Gamma_{e}} .
$$

Then, we set the product hilbert space $\mathbf{X}=X \times X$ and observe that $\mathbf{H}_{0}^{1}(\Omega)$ is the subspace of elements of $\mathbf{X}$ with zero jumps across all the internal edges inside $\Omega$.

Following the idea of taking a scalar value to handle the pressure on the whole domain $\Omega$, we construct $\mathbf{V}=\mathbf{X} \times \mathbb{R}$ and represent by $\underline{v}=(v, t)$ any element of $\mathbf{V}$ where $v \in \mathbf{X}$ and $t \in \mathbb{R}$. Obviously, $\mathbf{V}$ is Hilbert space with norm

$$
\|\underline{v}\|_{\mathbf{V}}^{2}=|v|_{\mathbf{X}}^{2}+t^{2} .
$$

Now for the pressure space we consider $\mathbf{M}=\prod_{s=1}^{S} L^{2}\left(\Omega^{s}\right)\left(\approx L^{2}(\Omega)\right)$ and represent the incompressibility restriction in terms of the continuous bilinear form $b: \mathbf{M} \times \mathbf{V} \mapsto \mathbb{R}$ given by

$$
b(q, \underline{v})=-\sum_{s=1}^{S}\left(q^{s}, \operatorname{div}\left(v^{s}\right)\right)_{\Omega^{s}}-t \sum_{s=1}^{S} \int_{\Omega^{s}} q^{s}
$$


where each $q^{s} \in L^{2}\left(\Omega^{s}\right)$. Next, for each $\Gamma_{e} \in \mathcal{E}_{0}$ we take $\mathbf{H}_{00}^{1 / 2}\left(\Gamma_{e}\right)=\left(H_{00}^{1 / 2}\left(\Gamma_{e}\right)\right)^{2}$, and handle the lagrange multipliers for the jumps with the space

$$
\mathbf{N}=\prod_{e=1}^{E} \mathbf{H}_{00}^{1 / 2}\left(\Gamma_{e}\right)
$$

endowed with the scalar product for all $\lambda, \mu \in \mathbf{N}$ given by

$$
(\lambda, \mu)_{\mathbf{N}}=\sum_{e=1}^{E}\left\{\lambda_{e}, \mu_{e}\right\}_{\Gamma_{e}} .
$$

A trivial characterization for $\mathbf{H}_{0}^{1}(\Omega)$ as a subspace of $\mathbf{X}$ is provided by the continuous bilinear form $c: \mathbf{N} \times \mathbf{X} \mapsto \mathbb{R}$ given by

$$
c(\lambda, v)=\sum_{e=1}^{E}\left\{\lambda_{e},[v]_{\Gamma_{e}}\right\}_{\Gamma_{e}}
$$

in the form

$$
\mathbf{H}_{0}^{1}(\Omega)=\{v \in \mathbf{X} ; c(\lambda, v)=0, \quad \forall \lambda \in \mathbf{N}\}
$$

The new formulation we propose at the continuous level is: find $\underline{u}=(u, \tau) \in \mathbf{V}, p=\left\{p^{s}\right\}_{s} \in \mathbf{M}$ and $\lambda=\left\{\lambda_{e}\right\}_{e} \in$ $\mathbf{N}$ such that

$$
\begin{gathered}
\sum_{s=1}^{S}\left(\nabla u^{s}, \nabla v^{s}\right)_{\Omega^{s}}+\sum_{e=1}^{E}\left\{[u]_{\Gamma_{e}},[v]_{\Gamma_{e}}\right\}_{\Gamma_{e}}+\tau t \\
-\sum_{s=1}^{S}\left(p^{s}, \operatorname{div}\left(v^{s}\right)\right)_{\Omega^{s}}-t \sum_{s=1}^{S} \int_{\Omega^{s}} p^{s}+\sum_{e=1}^{E}\left\{\lambda_{e},[v]_{\Gamma_{e}}\right\}_{\Gamma_{e}}=\sum_{s=1}^{S}\left(f, v^{s}\right)_{\Omega^{s}}, \\
-\sum_{s=1}^{S}\left(q^{s}, \operatorname{div}\left(u^{s}\right)\right)_{\Omega^{s}}-\tau \sum_{s=1}^{S} \int_{\Omega^{s}} q^{s}=0 \\
\sum_{e=1}^{E}\left\{\mu_{e},[u]_{\Gamma_{e}}\right\}_{\Gamma_{e}}=0
\end{gathered}
$$

for all $\underline{v}=(v, t) \in \mathbf{V}, q=\left\{q^{s}\right\}_{s} \in \mathbf{M}$ and $\mu=\left\{\mu_{e}\right\}_{e} \in \mathbf{N}$.

We see that we added the jumps to the elliptic terms to guarantee the control on the internal jumps and replaced the pairings $H_{00}^{-1 / 2}(\Gamma)-H_{00}^{1 / 2}(\Gamma)$ for the normal fluxes on the edges by the scalar product in $H_{00}^{1 / 2}(\Gamma)$, hence all terms are suitable to compute in a Galerkin approach.

Remark 2.3. The solution to this problem satisfies $u \in \mathbf{H}_{0}^{1}(\Omega), p \in L^{2}(\Omega)$ and $\tau=\int_{\Omega} p$; using any constant $q^{s} \equiv q^{\star}$ for all $s$ we also obtain $\tau=0$ and then $p \in L_{0}^{2}(\Omega)$. Finally, using test functions $v \in \mathbf{H}_{0}^{1}(\Omega)$, it gives the solution of the incompressible Stokes equations on $\Omega$.

In a more compact form, taking $F(\underline{v})=\sum_{s=1}^{S}\left(f, v^{s}\right)_{\Omega^{s}}$, the problem is

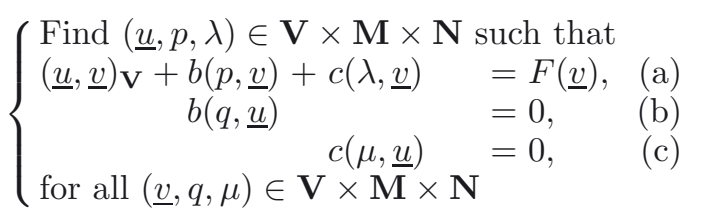


where

$$
\begin{aligned}
(\underline{u}, \underline{v})_{\mathbf{v}} & =\sum_{s=1}^{S}\left(\nabla u^{s}, \nabla v^{s}\right)_{\Omega^{s}}+\sum_{e=1}^{E}\left\{[u]_{\Gamma_{e}},[v]_{\Gamma_{e}}\right\}_{\Gamma_{e}}+\tau t, \\
b(p, \underline{v}) & =-\sum_{s=1}^{S}\left(p^{s}, \operatorname{div}\left(v^{s}\right)\right)_{\Omega^{s}}-t \sum_{s=1}^{S} \int_{\Omega^{s}} p^{s}, \\
c(\mu, \underline{u}) & =c(\mu, u)=\sum_{e=1}^{E}\left\{\mu_{e},[u]_{\Gamma_{e}}\right\}_{\Gamma_{e}} .
\end{aligned}
$$

The bilinear forms $b: \mathbf{M} \times \mathbf{V} \mapsto \mathbb{R}$ and $c: \mathbf{N} \times \mathbf{V} \mapsto \mathbb{R}$ are continuous with norms $\|b\| \leq 1$ and $\|c\| \leq 1$ and there exists positive constants $\beta>0$ and $\gamma>0$ such that

$$
\begin{aligned}
& \inf _{q \in \mathbf{M}} \sup _{\underline{v} \in \mathbf{V}} \frac{b(p, \underline{v})}{\|q\|_{\mathbf{M}}\|\underline{v}\|_{\mathbf{V}}} \geq \beta>0, \\
& \inf _{\mu \in \mathbf{N}} \sup _{\underline{v} \in \mathbf{V}} \frac{c(\mu, \underline{v})}{\|\mu\|_{\mathbf{N}}\|\underline{v}\|_{\mathbf{V}}} \geq \gamma>0 .
\end{aligned}
$$

Condition (2.12) was proved in Lemma 2.9 of [3] and in Theorem 2 in [6] and condition (2.11) follows from the global inf-sup condition (2.3).

Remark 2.4. A key result is that the inf-sup for the bilinear form $b$ is reached for a function $v \in \mathbf{V}$ with zero jumps, recall (2.3),

$$
\sup _{(v, t) \in V} \frac{b(p,(v, t))}{\|(v, t)\|_{V}} \geq \sup _{v \in \mathbf{H}_{0}^{1}(\Omega), t \in \mathbb{R}} \frac{b(p,(v, t))}{\left(\|\nabla v\|_{0, \Omega}^{2}+t^{2}\right)^{1 / 2}} \geq \beta\|p\|_{0, \Omega},
$$

while the inf-sup for bilinear form $c$ is reached for functions $v \in \mathbf{V}$ with non-zero jumps, see Lemma 2.9 of [3] for instance. As a consequence, formulation (2.10) has a unique solution that is the one of $(2.1)-(2.2)$.

\subsection{Setting of the dual problem}

Next, if we denote by primal variables velocity and pressure on the subdomains and dual variables the Lagrange multipliers, we eliminate the primal variables in terms of the dual variables, i.e., we obtain a dual problem that once solved will give the correct boundary data for the primal variables.

The writing of problem (2.10) in terms of operators will further simplify our analysis. For a Hilbert space $\mathbf{H}$, we denote by $(\cdot, \cdot)_{\mathbf{H}}$ its scalar product, by $\mathbf{H}^{\star}$ its dual space and represent its duality by $\langle\cdot, \cdot\rangle_{\mathbf{H}}$; moreover, for a linear operator $T$ we denote by $T^{\star}$ is adjoint. Then, (2.10) is

$$
\left\{\begin{aligned}
\text { Find }(\underline{u}, p, \lambda) \in \mathbf{V} & \times \mathbf{M} \times \mathbf{N} \text { such that } \\
A \underline{u}+B^{\star} p+C^{\star} \lambda & =F \text { in } \mathbf{V}^{\star} \\
B \underline{u} & =0 \text { in } \mathbf{M}^{\star} \\
C \underline{u} & =0 \text { in } \mathbf{N}
\end{aligned}\right.
$$

where $A$ is the Riesz isomorphism between $\mathbf{V}$ and $\mathbf{V}^{\star}$ that satisfies

$$
\langle A \underline{u}, \underline{v}\rangle_{\mathbf{V}}=(\underline{u}, \underline{v})_{\mathbf{V}}=\langle A \underline{v}, \underline{u}\rangle_{\mathbf{V}}, \quad \forall \underline{u}, \underline{v} \in \mathbf{V},
$$

$B$ is a linear operator from $\mathbf{V}$ into $\mathbf{M}^{\star}$ and

$$
\langle B \underline{v}, p\rangle_{\mathbf{M}}=b(p, \underline{v})=\left\langle B^{\star} p, \underline{v}\right\rangle_{\mathbf{v}} \quad \forall \underline{v} \in \mathbf{V}, \forall p \in \mathbf{M} .
$$


$C$ is a linear operator from $\mathbf{V}$ into $\mathbf{N}$ given by $C \underline{v}=\left([v]_{\Gamma_{e}}\right)_{e=1, \ldots, E}$. It can be characterized by

$$
(C \underline{v}, \lambda)_{\mathbf{N}}=c(\underline{v}, \lambda)=\left\langle C^{\star} \lambda, \underline{v}\right\rangle_{\mathbf{V}} \quad \forall(\underline{v}, \lambda) \in \mathbf{V} \times \mathbf{N} .
$$

Now we construct the dual problem for the variable $\lambda$, our dual variable by elimination of the primal variables. First

$$
\underline{u}=A^{-1} F-A^{-1} B^{\star} p-A^{-1} C^{\star} \lambda
$$

and using that $B \underline{u}=0$ and $C \underline{u}=0$ we obtain a system of two equations given by

$$
\begin{array}{ll}
\left(B A^{-1} B^{\star}\right) p+\left(B A^{-1} C^{\star}\right) \lambda=B A^{-1} F, & \text { in } \mathbf{M}^{\star}, \\
\left(C A^{-1} B^{\star}\right) p+\left(C A^{-1} C^{\star}\right) \lambda=C A^{-1} F, & \text { in } \mathbf{N} .
\end{array}
$$

The mapping $B A^{-1} B^{\star}$ is self-adjoint and positive definite thanks to the inf-sup condition (2.11) for $b$ we have

$$
\left\langle B A^{-1} B^{\star} p, p\right\rangle=\left\|B^{\star} p\right\|_{\mathbf{V}^{\star}}^{2} \geq \beta\|p\|_{\mathbf{M}}^{2} .
$$

As a consequence it has an inverse and allows to write $p$ in terms of $\lambda$

$$
p=\left(B A^{-1} B^{\star}\right)^{-1} B A^{-1} F-\left(B A^{-1} B^{\star}\right)^{-1}\left(B A^{-1} C^{\star}\right) \lambda .
$$

Finally, our dual problem for $\lambda$ is an equation on $\mathbf{N}$ that comes from the second equation (2.14) in the form:

$$
S \lambda=\ell,
$$

where $S$ and $\ell$ are

$$
\begin{aligned}
S & =\left(C A^{-1} C^{\star}\right)-\left(C A^{-1} B^{\star}\right)\left(B A^{-1} B^{\star}\right)^{-1}\left(B A^{-1} C^{\star}\right), \\
\ell & =\left(C A^{-1} F\right)-\left(C A^{-1} B^{\star}\right)\left(B A^{-1} B^{\star}\right)^{-1}\left(B A^{-1} F\right) .
\end{aligned}
$$

The proof of the following result can be seen in [7]

Theorem 2.5. Let be $S: \mathbf{N} \rightarrow \mathbf{N}$ defined as above. Then $S$ is a self-adjoint positive definite operator. That is to say

$$
(S \lambda, \mu)_{\mathbf{N}}=(S \mu, \lambda)_{\mathbf{N}} \quad \forall \mu, \lambda \in \mathbf{N},
$$

and there exists a constant $\sigma>0$ that only depends on the inf-sup conditions for bilinear forms $b$ and $c,(2.11)$ and (2.12) respectively, such that

$$
\sigma^{2}\|\lambda\|_{\mathbf{N}}^{2} \leq(S \lambda, \lambda)_{\mathbf{N}} \leq\|\lambda\|_{\mathbf{N}}^{2}, \quad \forall \lambda \in \mathbf{N},(\lambda \neq 0) .
$$

As a consequence, our dual problem (2.15) is well posed and has a unique solution that gives the correct Lagrange multipliers for (2.10).

Remark 2.6. If we consider the bilinear form

$$
\mathcal{B}(\underline{u}, p ; \underline{v}, q)=(\underline{u}, \underline{v})_{\mathbf{V}}+b(p, \underline{v})-b(q, \underline{u}),
$$

an inf-sup condition for the variable $(\underline{u}, p) \in \mathbf{V} \times \mathbf{M}$ of the form

$$
\sup _{(\underline{v}, q) \neq 0} \frac{\mathcal{B}(\underline{u}, p ; \underline{v}, q)}{\|\underline{v}\|_{\mathbf{V}}+\|q\|_{M}} \geq \sigma\left(\|\underline{v}\|_{\mathbf{V}}+\|q\|_{M}\right), \quad \forall(\underline{u}, p) \in \mathbf{V} \times M
$$

would aswell allow the construction of a well-posed dual problem. This is better suited for stabilization methods. 
Formulation (2.15), or (2.10), is equivalent to (2.1)-(2.2) and we obtain the following theorem.

Theorem 2.7. $(\underline{u}, p, \lambda) \in \mathbf{V} \times \mathbf{M} \times \mathbf{N}$ is the unique solution for (2.10) or (2.15) if and only if $\underline{u}=(u, 0)$ with $u \in \mathbf{H}_{0}^{1}(\Omega)$ and $p \in L_{0}^{2}(\Omega)$ are the unique solution for problem $(2.1)-(2.2)$. Moreover, $\lambda \in \mathbf{N}$ given by $\lambda=\left(\lambda_{e}\right)_{e}$ with $\lambda_{e} \in \mathbf{H}_{00}^{1 / 2}\left(\Gamma_{e}\right)$ is the Riesz representation of $\pm\left(-\partial_{n_{e}} u+p n_{e}\right) \in\left(H_{00}^{-1 / 2}\left(\Gamma_{e}\right)\right)^{2}$ for all $\Gamma_{e} \in \mathcal{E}_{0}$.

Proof. Let $(\underline{u}, p, \lambda) \in \mathbf{V} \times \mathbf{M} \times \mathbf{N}$ be the unique solution obtained using (2.15) or equivalently (2.10). Then, due to $(2.10(\mathrm{c}))$, all the jumps of $u$ must vanish and therefore $u \in \mathbf{H}_{0}^{1}(\Omega)$ and it is straightforward to check that $(u, p)$ is the solution of the incompressible Stokes problem. Next, as each $\Omega^{s}$ is a polygonal domain we consider the subspace $\mathbf{W}^{s}$ of $\mathbf{H}^{1}\left(\Omega^{s}\right)$ given by

$$
\mathbf{W}^{s}=\left\{v \in\left(H^{1}\left(\Omega^{s}\right) \cap H_{0}^{1}(\Omega)\right)^{2} ; v_{\left.\right|_{e}} \in \mathbf{H}_{00}^{1 / 2}\left(\Gamma_{e}\right), \Gamma_{e} \subset \partial \Omega^{s} \cap \mathcal{E}_{0}\right\}
$$

and for each $v^{s} \in \mathbf{W}^{s}$ we take $\widetilde{v^{s}} \in \mathbf{X}$ the extension by zero of $v^{s}$ outside $\Omega^{s}$ and use it into (2.10(a)). Then by Green's formula on polygonal domains, see Grisvard [11], we recover the result about the normal fluxes.

\section{StabiLized APPROXIMATION}

In [7] the discrete approximation using a stable pair of finite element spaces was considered. Now we reduce the number of degrees of freedom needed by means of stabilization techniques. We propose to stabilize the discrete Stokes problem via the usual Brezzi-Pitkaranta approach, see [9] for instance, because the main ideas can be extended to more elaborated stabilization techniques.

To simplify we consider a conforming triangulation $\mathcal{T}_{h}$ of $\bar{\Omega}$ that contains the skeleton $\mathcal{E}_{0}$ as union of edges of triangles and so that on each edge the same partition is inherited from both sides. As usual, $h$ is the mesh size, i.e., $h=\max h_{\kappa}$ where $\kappa$ is a generic element of the mesh and $h_{\kappa}$ is the longest side of $\kappa$. As $\mathcal{T}_{h}$ is also compatible with the subdivision of $\Omega$, its restriction to each $\overline{\Omega_{s}}$ gives a mesh $\mathcal{T}_{h}^{s}$ on $\overline{\Omega^{s}}$.

We use the standard $\mathbb{P}_{1}$ finite element for velocity and pressure on each subdomain. Define the family of subspaces $\left\{Y_{h}\right\}_{h} \subset H_{0}^{1}(\Omega)$ and $\left\{Q_{h}\right\}_{h} \subset H^{1}(\Omega)$ given by

$$
\begin{aligned}
Y_{h} & =\left\{v \in H_{0}^{1}(\Omega) ; v_{\left.\right|_{\kappa}} \in \mathbb{P}_{1}(\kappa), \forall \kappa \in \mathcal{T}_{h}\right\}, \\
Q_{h} & =\left\{p \in H^{1}(\Omega) ; p_{\left.\right|_{\kappa}} \in \mathbb{P}_{1}(\kappa), \forall \kappa \in \mathcal{T}_{h}\right\}
\end{aligned}
$$

where $\mathbb{P}_{r}(\kappa)$ is the space of polynomials of degree less or equal to $r$ in the two variables $x$ and $y$. On each subdomain, we take also

$$
Y_{h}\left(\Omega^{s}\right)=Y_{h} \cap H^{1}\left(\Omega^{s}\right), \quad Q_{h}\left(\Omega^{s}\right)=Q_{h} \cap H^{1}\left(\Omega^{s}\right), s \leq S .
$$

Remark 3.1. As $\Omega \subset \mathbb{R}^{2}$, we have $Y_{h}, Q_{h} \subset C^{0}(\bar{\Omega})$ for any $h>0$.

Consider now $\mathbf{X}_{h}=X_{h} \times X_{h}$, where $X_{h}$ is the broken version of $Y_{h}$ given by

$$
\begin{aligned}
X_{h}= & \left\{v \in L^{2}(\Omega) ; v^{s} \in Y_{h}\left(\Omega^{s}\right), \forall s=1,2, \ldots, S,\right. \\
& \text { and } v \text { is continuous at every cross point in } \mathcal{C}\} \subset X,
\end{aligned}
$$

define $\mathbf{V}_{h}=\mathbf{X}_{h} \times \mathbb{R}, \mathbf{M}_{h}=\prod_{s=1}^{S} Q_{h}\left(\Omega^{s}\right)$ and finally $\mathbf{N}_{h} \subset \mathbf{N}$ given by the restriction of functions in $\mathbf{X}_{h}$ to the skeleton $\mathcal{E}_{0}$.

For the stabilization approach to Stokes problem the inf-sup condition is achieved on the coupled velocitypressure pair. We follow this idea to construct the dual problem now. Set the bilinear form

$$
\mathcal{B}_{h}\left(\underline{u}_{h}, p_{h} ; \underline{v}_{h}, q_{h}\right)=\left(\underline{u}_{h}, \underline{v}_{h}\right)_{\mathbf{V}}+b\left(p_{h}, \underline{v}_{h}\right)-b\left(q_{h}, \underline{u}_{h}\right)+R_{h}\left(p_{h}, q_{h}\right),
$$


where $R_{h}$ is the standard Brezzi-Pitkaranta stabilization term applied to each subdomain

$$
R_{h}\left(p_{h}, q_{h}\right)=\sum_{s=1}^{S} \alpha^{s} \sum_{\kappa \in \mathcal{T}_{h}^{s}} h_{\kappa}^{2}\left(\nabla p_{h}^{s}, \nabla q_{h}^{s}\right)_{\kappa}
$$

and let $F\left(\underline{v}_{h}\right)=\sum_{s=1}^{S}\left(f, v_{h}^{s}\right)_{\Omega^{s}}$. Then, our problem is

$$
\left\{\begin{array}{l}
\text { Find }\left(\underline{u}_{h}, p_{h}, \lambda_{h}\right) \in \mathbf{V}_{h} \times \mathbf{M}_{h} \times \mathbf{N}_{h} \text { such that } \\
\mathcal{B}_{h}\left(\underline{u}_{h}, p_{h} ; \underline{v}_{h}, q_{h}\right)+c\left(\lambda_{h}, \underline{v}_{h}\right)=\quad F\left(\underline{v}_{h}\right), \\
\quad c\left(\mu_{h}, \underline{u}_{h}\right)=0, \\
\text { for all }\left(\underline{v}_{h}, q_{h}, \mu_{h}\right) \in \mathbf{V}_{h} \times \mathbf{M}_{h} \times \mathbf{N}_{h} .
\end{array}\right.
$$

With the obvious notation, we also have

$$
\left\{\begin{array}{l}
\text { Find }\left(\underline{u}_{h}, p_{h}, \lambda_{h}\right) \in \mathbf{V}_{h} \times \mathbf{M}_{h} \times \mathbf{N}_{h} \text { such that } \\
\mathcal{B}_{h}\left(\underline{u}_{h}, p_{h}\right)+\mathcal{C}^{\star} h\left(\lambda_{h}\right)=F \\
\mathcal{C}_{h}\left(\underline{u}_{h}, p_{h}\right)=0 .
\end{array}\right.
$$

Provided $\mathcal{B}_{h}$ invertible we will be able to eliminate the unknows $\left(\underline{u}_{h}, p_{h}\right)$ and construct the dual problem for the Lagrange multplier $\lambda_{h}$

$$
\left(\mathcal{C}_{h} \mathcal{B}_{h}^{-1} \mathcal{C}_{h}\right) \lambda_{h}=\mathcal{C}_{h} \mathcal{B}_{h}^{-1} F
$$

Finally, by proving a uniform discrete inf-sup condition for the bilinear forms $\mathcal{B}_{h}$ and $\mathcal{C}_{h}$, this dual problem is a symmetric positive definite problem that can be solved by Conjugate Gradient without preconditioning and with a mesh independent rate of convergence.

The discrete uniform inf-sup condition for $\mathcal{C}_{h}$ on the pair $\mathbf{V}_{h}$ and $\mathbf{N}_{h}$ is a well known result, see for instance Theorem 4 in [4]:

Lemma 3.2. There exists a positive constant $\gamma>0$ such that for all $\mu_{h} \in \mathbf{N}_{h}$

$$
\sup _{\left(v_{h}, t\right) \in \mathbf{V}_{\mathbf{h}}} \frac{c\left(\mu_{h},\left(v_{h}, t\right)\right)}{\left\|\left(v_{h}, t\right)\right\|_{\mathbf{V}}} \geq \sup _{v_{h} \in \mathbf{X}_{h}} \frac{c\left(\mu_{h}, v_{h}\right)}{\left\|v_{h}\right\|_{\mathbf{X}}} \geq \gamma\left\|\mu_{h}\right\|_{\mathbf{N}} .
$$

This result can be obtained using the standard finite element extension theorems or using the extra regularity of solutions for elliptic problems in polygonal domains.

Secondly, we check the inf-sup condition for $\mathcal{B}_{h}$. This is more delicated and we combine the standard proof of stability for the Brezzi-Pitkaranta method, see [9], with the technique to reduce the global inf-sup stability bound to a local ones used in [10], Theorem 1.12, page 130. This is the main theoretical result of this work.

Theorem 3.3. There exists a positive constant $\gamma>0$ independent of the mesh size such that

$$
\sup _{\underline{v}_{h} \in \mathbf{V}_{\mathbf{h}}} \frac{\mathcal{B}_{h}\left(\underline{u}_{h}, p_{h} ; \underline{v}_{h}, q_{h}\right)}{\left\|\underline{v}_{h}\right\|_{\mathbf{V}}+\left\|q_{h}\right\|_{M}} \geq \gamma\left\{\left\|\underline{u}_{h}\right\|_{\mathbf{v}}+\left\|p_{h}\right\|_{M}\right\}
$$

and the function $v_{h} \in \mathbf{X}_{h}$ that gives the maximum satisfies $v_{h} \in C^{0}(\bar{\Omega})$, i.e., its jumps across all interfaces are all zero.

Proof. First, we see that using $\underline{u}_{h}=\left(u_{h}, \tau\right) \in \mathbf{X}_{h} \times \mathbb{R}$ we have

$$
\mathcal{B}\left(\underline{u}_{h}, p_{h} ; \underline{u}_{h}, p_{h}\right)=\left\|u_{h}\right\|_{\mathbf{X}}^{2}+\tau^{2}+\sum_{s=1}^{S} \alpha^{s} \sum_{\kappa \in \mathcal{T}_{h}^{s}} h_{\kappa}^{2}\left\|\nabla p_{h}^{s}\right\|_{0, \kappa}^{2}
$$


and second that for any $\underline{v}_{h}=\left(v_{h}, t\right)$ we have

$$
\mathcal{B}\left(\underline{u}_{h}, p_{h} ; \underline{v}_{h}, 0\right)=\left(u_{h}, v_{h}\right) \mathbf{x}+\tau t-\sum_{s=1}^{S}\left(p_{h}^{s}, \operatorname{div}\left(v_{h}^{s}\right)\right)_{\Omega^{s}}-t \sum_{s=1}^{S} \int_{\Omega^{s}} p_{h}^{s} .
$$

Our goal will be to find $v_{h}^{\star}=\sum_{s=1}^{S} v_{h}^{s} \in \mathbf{X}_{h} \cap C^{0}(\Omega)$ that allows to control the difference $p_{h}-\bar{p}_{h} \in \mathbf{M}_{h} \cap L_{0}^{2}(\Omega)$ as in the continuous case. Then we would take

$$
t^{\star}=-\bar{p}_{h}=-\frac{1}{|\Omega|} \int_{\Omega} p_{h},
$$

as previously, using the stabilization term to control the defect that appears on the inf-sup estimate for the pair $\mathbb{P}_{1}-\mathbb{P}_{1}$. With that in mind set

$$
\pi_{h}=p_{h}-\bar{p}_{h} \in \mathbf{M}_{h} \cap L_{0}^{2}(\Omega)
$$

and observe that

$$
\pi_{h}=\tilde{\pi}_{h}+\bar{\pi}_{h}
$$

where $\tilde{\pi}_{h}^{s}$, the restriction to each $\Omega^{s}$ of $\tilde{\pi}_{h}$, is a $\mathbb{P}_{1}$ finite element function that belongs to $L_{0}^{2}\left(\Omega^{s}\right) \cap C^{0}\left(\Omega^{s}\right)$ and $\bar{\pi}_{h}$ is constant on $\Omega^{s}$ such that globally $\bar{\pi}_{h} \in L_{0}^{2}(\Omega)$; this is well known to be an orthogonal decomposition. As a consequence, see [9], there exists $\tilde{w}_{h}^{s} \in Y\left(\Omega^{s}\right) \cap\left(H_{0}^{1}\left(\Omega^{s}\right)\right)^{2}$ such that

$$
\begin{aligned}
\frac{\left(\tilde{\pi}_{h}^{s}, \operatorname{div}\left(\tilde{w}_{h}^{s}\right)\right)}{\left\|\nabla \tilde{w}_{h}^{s}\right\|_{0, \Omega^{s}}} & \geq c_{1}^{s}\left\|\tilde{\pi}_{h}^{s}\right\|_{0, \Omega^{s}}-c_{2}^{s}\left(\sum_{\kappa \in \mathcal{T}_{h}^{s}} h_{\kappa}^{2}\left\|\nabla \tilde{\pi}_{h}^{s}\right\|_{0, \kappa}^{2}\right)^{1 / 2} \\
& =c_{1}^{s}\left\|\tilde{\pi}_{h}^{s}\right\|_{0, \Omega^{s}}-c_{2}^{s}\left(\sum_{\kappa \in \mathcal{T}_{h}^{s}} h_{\kappa}^{2}\left\|\nabla p_{h}^{s}\right\|_{0, \kappa}^{2}\right)^{1 / 2}
\end{aligned}
$$

and such that

$$
\left\|\nabla \tilde{w}_{h}^{s}\right\|_{0, \Omega^{s}}=\left\|\tilde{\pi}_{h}^{s}\right\|_{0, \Omega^{s}}
$$

for some mesh independent constants $c_{1}^{s}, c_{2}^{s}$. Define now the function on $\Omega$ (bubble function on each $\Omega^{s}$ ) given by $\tilde{w}_{h}=\sum_{s=1}^{S} \tilde{w}_{h}^{s}$ and take $c_{i}=\min _{s}\left\{c_{i}^{s}\right\}$ for $i=1,2$, then

$$
\begin{aligned}
\left(\tilde{\pi}_{h}, \operatorname{div}\left(\tilde{w}_{h}\right)\right)_{0, \Omega} & =\sum_{s=1}^{S}\left(\tilde{\pi}_{h}^{s}, \operatorname{div}\left(\tilde{w}_{h}^{s}\right)\right)_{0, \Omega^{s}} \\
& \geq c_{1}\left\|\tilde{\pi}_{h}\right\|_{0, \Omega}^{2}-c_{2} \sum_{s=1}^{S}\left\|\tilde{\pi}_{h}^{s}\right\|_{0, \Omega^{s}}^{2}\left(\sum_{\kappa \in \mathcal{T}_{h}^{s}} h_{\kappa}^{2}\left\|\nabla p_{h}^{s}\right\|_{0, \kappa}^{2}\right)^{1 / 2} .
\end{aligned}
$$

Standard application of Young's inequality gives for some new $c_{1}$ and $c_{2}$ that

$$
\left(\tilde{\pi}_{h}, \operatorname{div}\left(\tilde{w}_{h}\right)\right)_{0, \Omega} \geq c_{1}\left\|\tilde{\pi}_{h}\right\|_{0, \Omega}^{2}-c_{2} \sum_{s=1}^{S} \sum_{\kappa \in \mathcal{T}_{h}^{s}} h_{\kappa}^{2}\left\|\nabla p_{h}^{s}\right\|_{0, \kappa}^{2} .
$$


On the other hand, for the function $\bar{\pi}_{h}$ that is constant on each $\Omega^{s}$ and with the only restriction of $\bar{\pi}_{h} \in L_{0}^{2}(\Omega)$ we have enough degrees of freedom to construct $z_{h} \in \mathbf{X}_{h} \cap\left(C^{0}(\Omega)\right)^{2}$ such that

$$
\left(\operatorname{div}\left(z_{h}\right), \bar{\pi}_{h}\right)_{\Omega}=\left\|\bar{\pi}_{h}\right\|_{0 \Omega}^{2}, \quad\left\|\nabla z_{h}\right\|_{0, \Omega}=\left\|\bar{\pi}_{h}\right\|_{0, \Omega} .
$$

Take then, for a real parameter $\beta$ to be fixed, the function

$$
w_{h}=\tilde{w}_{h}+\beta z_{h} \in \mathbf{X}_{h} \cap\left(C^{0}(\Omega)\right)^{2} .
$$

Then, it is standard that for $\beta>0$ small enough we obtain

$$
\left(\pi_{h}, \operatorname{div}\left(w_{h}\right)\right)_{0, \Omega} \geq c_{1}\left\|\pi_{h}\right\|_{0, \Omega}^{2}-c_{2} \sum_{s=1}^{S} \sum_{\kappa \in \mathcal{T}_{h}^{s}} h_{\kappa}^{2}\left\|\nabla p_{h}^{s}\right\|_{0, \kappa}^{2} .
$$

with

$$
\left\|\nabla w_{h}\right\|_{0, \Omega} \leq\left\|\pi_{h}\right\|_{0, \Omega} .
$$

Finally, we take $\underline{w}_{h}=\left(w_{h}, t^{\star}\right)$ with $t^{\star}=|\Omega|^{-1} \int_{\Omega} p_{h}$; then, using again Young's inequality whenever needed,

$$
\begin{aligned}
\mathcal{B}_{h}\left(\underline{u}_{h}, p_{h} ;-\underline{w}_{h}, 0\right)= & -\sum_{s=1}^{S}\left(\nabla u_{h}^{s}, \nabla w_{h}^{s}\right)_{0, \Omega^{s}}-\tau|\Omega|^{-1} \int_{\Omega^{s}} p_{h} \\
& +\sum_{s=1}^{S}\left(p_{h}^{s}, \operatorname{div}\left(w_{h}^{s}\right)\right)_{\Omega^{s}}+|\Omega|^{-1}\left(\int_{\Omega^{s}} p_{h}\right)^{2} \\
\geq & \frac{-1}{\epsilon} \sum_{s=1}^{S}\left\|\nabla u_{h}^{s}\right\|_{0, \Omega^{s}}^{2}-\epsilon \sum_{s=1}^{S}\left\|\nabla w_{h}^{s}\right\|_{0, \Omega^{s}}^{2}+\frac{-1}{\epsilon} \tau^{2}-\epsilon \bar{p}_{h}^{2} \\
& +c_{1}\left\|\pi_{h}\right\|_{0, \Omega}^{2}-c_{2} \sum_{s=1}^{S} \sum_{\kappa \in \mathcal{T}_{h}^{s}} h_{\kappa}^{2}\left\|\nabla p_{h}^{s}\right\|_{0, \kappa}^{2}+|\Omega| \bar{p}_{h}^{2} \\
\geq & \frac{-1}{\epsilon}\left\{\sum_{s=1}^{S}\left\|\nabla u_{h}^{s}\right\|_{0, \Omega^{s}}^{2}+\tau^{2}\right\}-\epsilon\left\|\pi_{h}\right\|_{0, \Omega}^{2}+\{|\Omega|-\epsilon\} \bar{p}_{h}^{2} \\
& +c_{1}\left\|\pi_{h}\right\|_{0, \Omega}^{2}-c_{2} \sum_{s=1}^{S} \sum_{\kappa \in \mathcal{T}_{h}^{s}} h_{\kappa}^{2}\left\|\nabla p_{h}^{s}\right\|_{0, \kappa}^{2}
\end{aligned}
$$

then, for $\epsilon \leq \epsilon_{0}$ we have

$$
\begin{aligned}
\mathcal{B}_{h}\left(\underline{u}_{h}, p_{h} ;-\underline{w}_{h}, 0\right) \geq & \frac{-1}{\epsilon}\left\{\sum_{s=1}^{S}\left\|\nabla u_{h}^{s}\right\|_{0, \Omega^{s}}^{2}+\tau^{2}\right\}+c_{3}|\Omega| \bar{p}_{h}^{2} \\
& +c_{1}\left\|p_{h}-\bar{p}_{h}\right\|_{0, \Omega}^{2}-c_{2} \sum_{s=1}^{S} \sum_{\kappa \in \mathcal{T}_{h}^{s}} h_{\kappa}^{2}\left\|\nabla p_{h}^{s}\right\|_{0, \kappa}^{2} \\
\geq & \frac{-1}{\epsilon}\left\{\left\|u_{h}\right\|_{\mathbf{X}}^{2}+\tau^{2}\right\}+c_{1}\left\|p_{h}\right\|_{0, \Omega}^{2}-c_{2} \sum_{s=1}^{S} \sum_{\kappa \in \mathcal{T}_{h}^{s}} h_{\kappa}^{2}\left\|\nabla p_{h}^{s}\right\|_{0, \kappa}^{2} .
\end{aligned}
$$

Finally, for $\delta>0$ to be fixed,

$$
\left(\underline{v}_{h}, q_{h}\right)=\left(\underline{u}_{h}-\delta \underline{w}_{h}, p_{h}\right)
$$


gives

$$
\begin{aligned}
\mathcal{B}_{h}\left(\underline{u}_{h}, p_{h} ; \underline{v}_{h}, q_{h}\right) \geq & \left\|u_{h}\right\|_{\mathbf{X}}^{2}+\tau^{2}+\sum_{s=1}^{S} \alpha^{s} \sum_{\kappa \in \mathcal{T}_{h}^{s}} h_{\kappa}^{2}\left\|\nabla p_{h}^{s}\right\|_{0, \kappa}^{2} \\
& +\frac{-\delta}{\epsilon}\left\{\left\|u_{h}\right\|_{\mathbf{X}}^{2}+\tau^{2}\right\}+\delta c_{1}\left\|p_{h}\right\|_{0, \Omega}^{2}-\delta c_{2} \sum_{s=1}^{S} \sum_{\kappa \in \mathcal{T}_{h}^{s}} h_{\kappa}^{2}\left\|\nabla p_{h}^{s}\right\|_{0, \kappa}^{2} \\
= & \left\{1-\frac{\delta}{\epsilon}\right\}\left\{\left\|u_{h}\right\|_{\mathbf{X}}^{2}+\tau^{2}\right\}+\delta c_{1}\left\|p_{h}\right\|_{0, \Omega}^{2} \\
& +\sum_{s=1}^{S}\left(\alpha^{s}-\delta c_{2}\right) \sum_{\kappa \in \mathcal{T}_{h}^{s}} h_{\kappa}^{2}\left\|\nabla p_{h}^{s}\right\|_{0, \kappa}^{2} .
\end{aligned}
$$

As a consequence, if we take $\delta>0$ such that

$$
1-\delta \epsilon^{-1}>0, \quad \alpha^{s}-\delta c_{2}>0, s=1,2, \ldots, S
$$

we obtain the existence of $\gamma>0$ independent of $h$ and the $\alpha^{s}$ such that

$$
\mathcal{B}_{h}\left(\underline{u}_{h}, p_{h} ; \underline{v}_{h}, q_{h}\right) \geq \gamma\left\{\left\|\underline{u}_{h}\right\|_{\mathbf{V}}^{2}+\left\|p_{h}\right\|_{M}^{2}\right\} .
$$

Moreover, tracking back our construction of $\underline{v}_{h}$ we find

$$
\left\|\underline{v}_{h}\right\|_{\mathbf{V}}^{2} \leq\left\|\underline{u}_{h}\right\|_{\mathbf{V}}^{2}+\delta\left\|\underline{w}_{h}\right\|_{\mathbf{V}}^{2} \leq C\left(\left\|\underline{u}_{h}\right\|_{\mathbf{X}}^{2}+\left\|p_{h}\right\|_{0, \Omega}^{2}\right)
$$

for a constant $C>0$ also independent of $h$ that yields our discrete stability condition for $\mathcal{B}_{h}$.

Next, we cast the problem in terms of solving an equation for the dual discrete variable $\lambda_{h}$ set on the Lagrange multiplier space $\mathbf{N}_{h}$. The equation is

$$
S_{h} \lambda_{h}=\ell_{h}
$$

where the operator $S_{h}$ is SPD with a uniformly bounded spectrum

$$
\sigma^{2}\|\mu\|_{\mathbf{N}}^{2} \leq\left(S_{h} \mu, \mu\right)_{\mathbf{N}} \leq\|\mu\|_{\mathbf{N}}^{2}, \quad \forall \mu \in \mathbf{N}_{h}
$$

thanks to the uniform discrete inf-sup conditions. As a consequence, we solve (3.5) via an external cycle performed by the Conjugate Gradient Method (CG) without preconditioner in an h independent number of steps. This external cycle has associated an internal cycle for the computation of the action of $S_{h}$ on a CG direction. This internal problem is computed also via a preconditioned Conjugate Gradient, using independent subdomain solves, see [7] for a more detailed explanation. To sum up, we have

1. an external computational cycle, the CG for $S$ with a fixed number of iterations independent of the discretization parameter $h$; and

2. at each iteration of this external cycle, the resolution of a primal problem of the form:

Find $\left(\underline{w}_{h}, q_{h}\right) \in \mathbf{V}_{h} \times \mathbf{M}_{h}$ such that

$$
\begin{aligned}
A \underline{w}_{h}+B^{\star} q_{h} & =\xi \text { in } \mathbf{V}_{h}, \\
B \underline{w}_{h} & =0 \text { in } \mathbf{M}_{h}
\end{aligned}
$$

where for the initial residuous $r_{0}$ we have $\xi=F$ and for the iteration $m \geq 0$ we have $\xi=C^{\star} d_{m}$. 

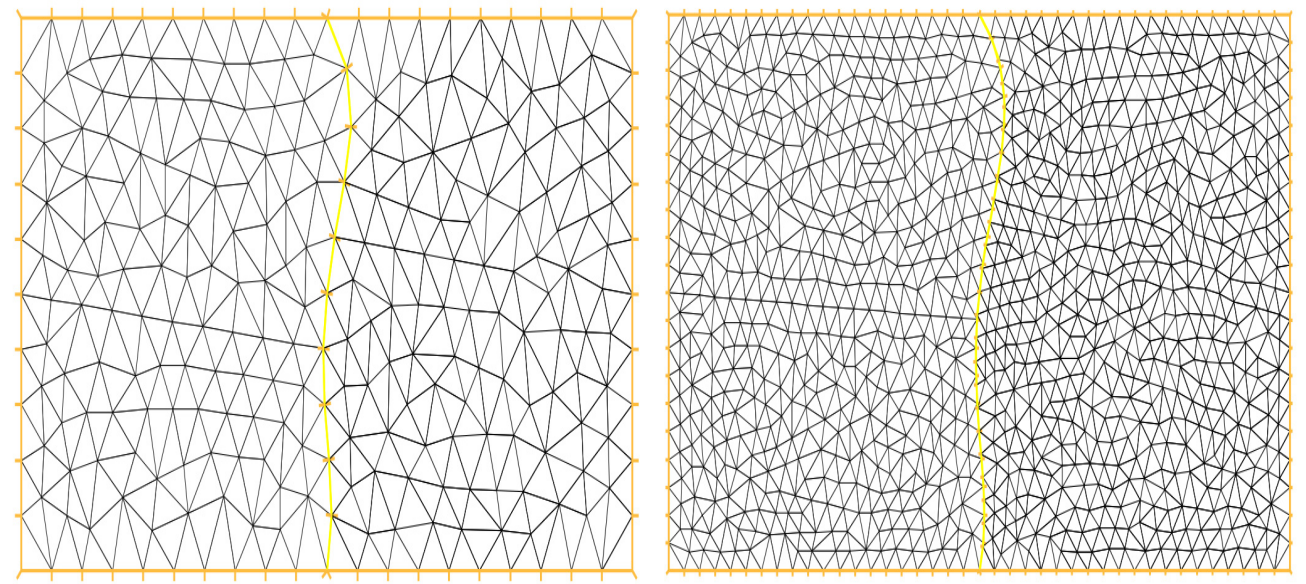

Figure 2. Triangulations for the two subdomain test with conforming and nonconforming meshes, curved interface.

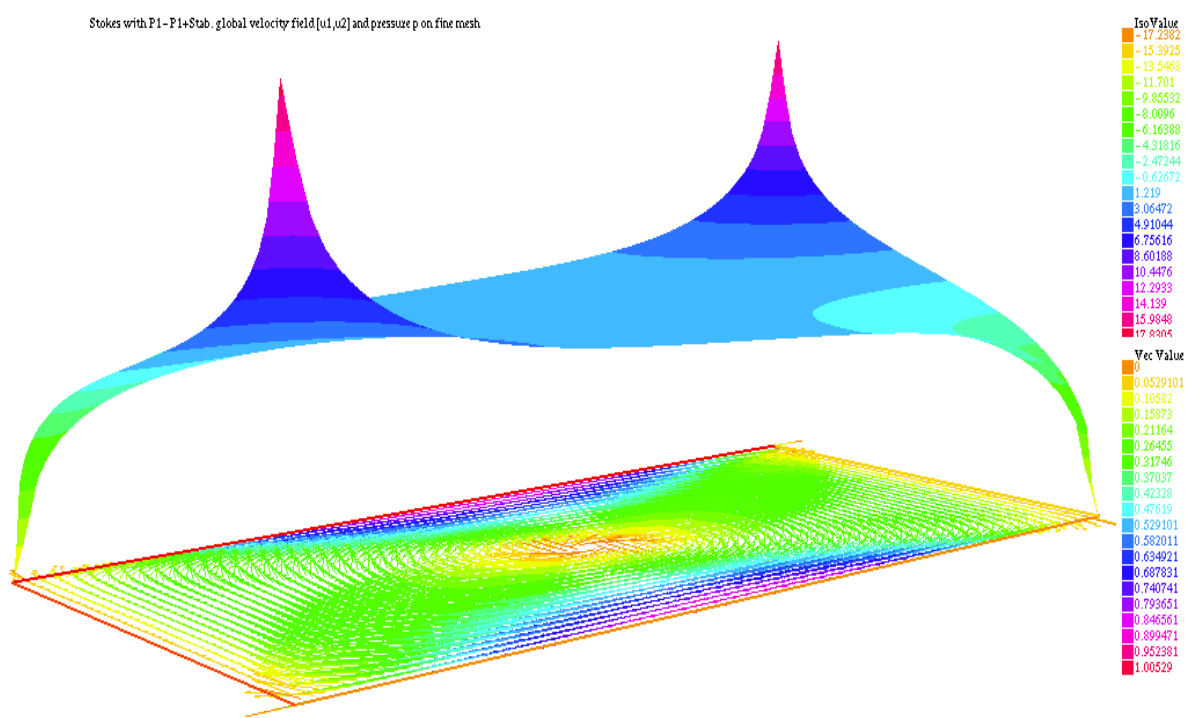

Figure 3. Global solution on fine mesh, pressure and velocity fields for first test.

\section{SOME NUMERICAL TESTS}

All the numerical tests that we present have been performed with FreeFem $++[8]$.

\subsection{Academic test 1: two subdomains}

We first consider $\Omega=(0,2) \times(0,1)$ and split it into two subdomains through a curved interface using conforming and nonconforming meshes; on Figure 2 we show a typical conforming and nonconforming mesh configuration in the case $h=1 / 10$, in the nonconforming. We move the flow via the boundary conditions

$$
u(x, 1)=x(2-x), \quad u(x, 0)=-x(2-x), \quad u(0, y)=u(2, y)=0
$$

which somehow resemble the driven lid test and Figure 3 shows the computed solution in the global domain. On Figure 4 we show the decay of the residuals for the CG method measure in the $H_{00}^{1 / 2}$ norm while on Figure 5 

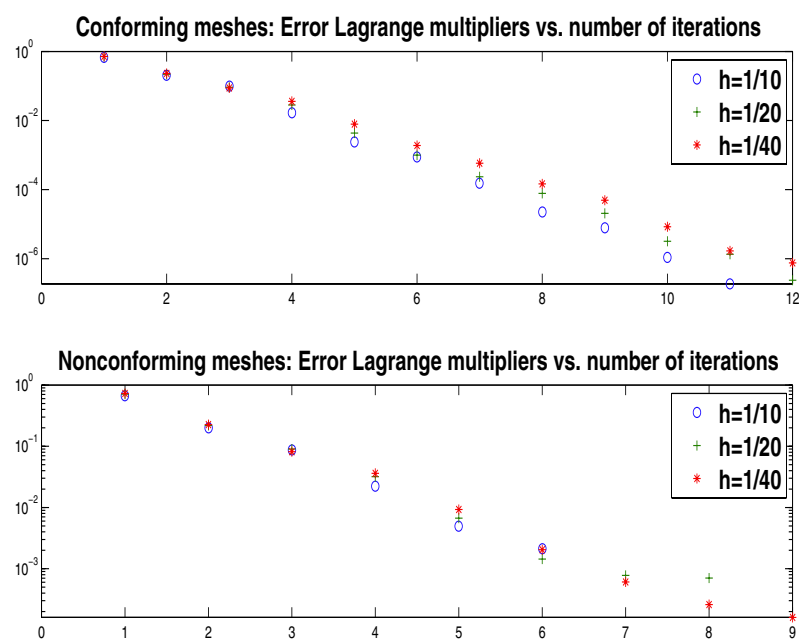

FiguRE 4. Log in base 10 plot of the residuals for the CG method with respect to the number of iterations. Residuals measured in the $H_{00}^{1 / 2}$ norm for first test and with different values of the mesh size $h$. In the nonconforming case the resolution of the interface from both subdomains is different but similar.
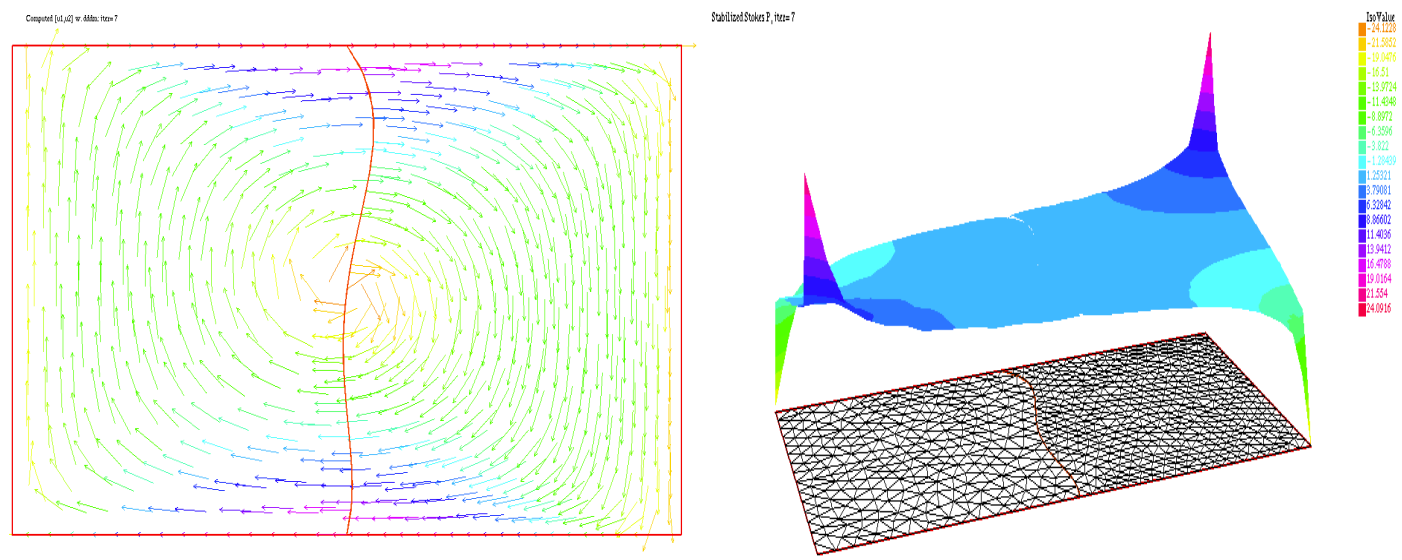

FIGURE 5. Computed velocity field and pressure for test 1 with non the conforming mesh shown on Figure 2.

we show the computed solution via our domain decomposition method. We have found similar results for the conforming and non conforming configuration.

\subsection{Academic test 2: a floating subdomain}

As a second and final test we consider a disk $\Omega$ centered at $(0,0)$ with radius $R=2$ with a floating subdomain, another disk centered at the point $(0.5,25)$ with radius $r=0.75$. In this last configuration there are no cross points and we just work with the $H^{1 / 2}$ scalar product on the internal disk boundary. We let the flow rotate via 


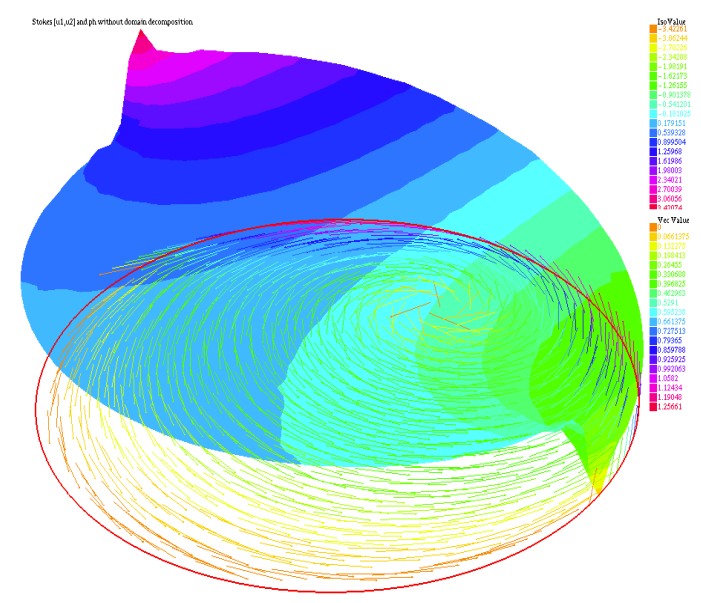

FiguRE 6. Global pressure and velocity solution for second test.

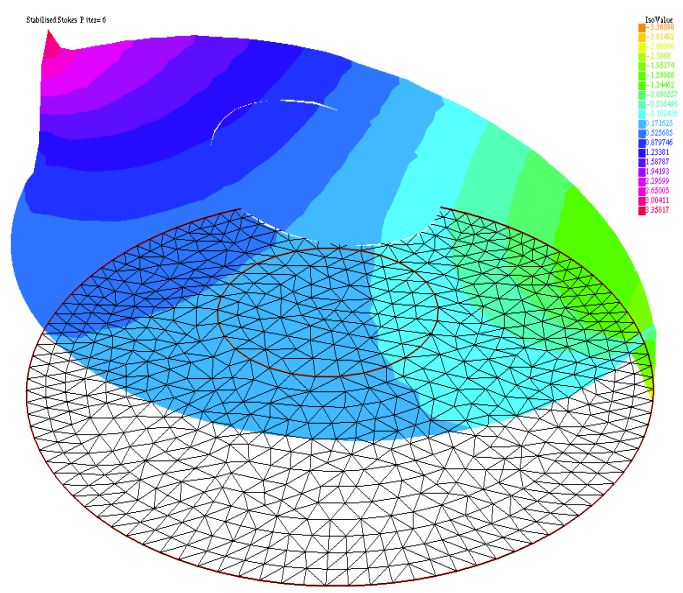

FiguRE 7. Computed pressure solution for second test with conforming triangulations.

the boundary conditions

$$
\begin{aligned}
& u(x, y)=-x y / 2, \quad v(x, y)=x / 2, \text { on } \Gamma_{1}=\{(x, y) \in \partial \Omega, \quad x>0\} \\
& u(x, y)=0, \quad v(x, y)=0 \quad \text { on } \quad \partial \Omega \backslash \Gamma_{1} .
\end{aligned}
$$

Figures 6 to 8 show the global and computed solution with conforming triangulations after six iterations, here the convergence results and ratios are similar to the previous example. To conclude, Figures 9 to 11 show the results using nonconforming triangulations after two iterations.

\section{Conclusions}

The use of the $H_{00}^{1 / 2}$ norm allows to obtain an abstract framework for a FETI-DP mortar method which is simple to analyze in continuous and in discrete formulation. We studied the use of this tool on several differential 


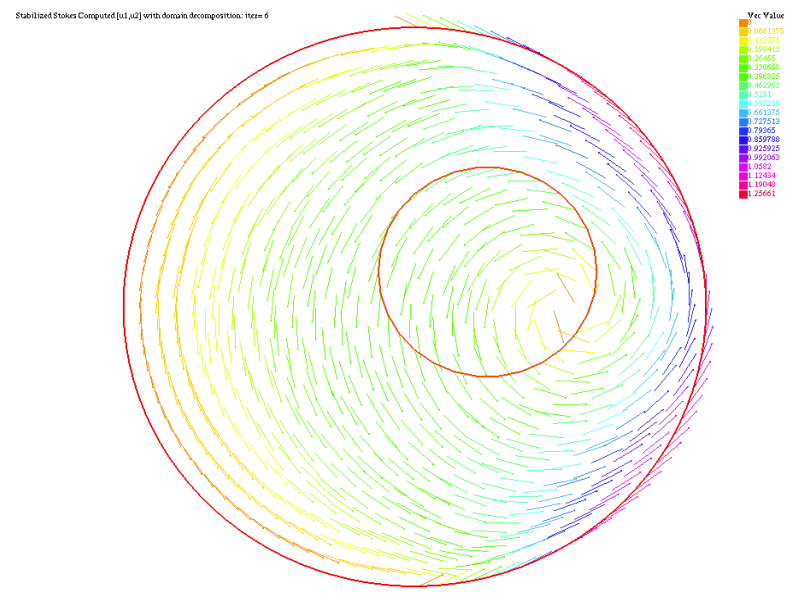

Figure 8. Computed velocity field solution for second test with conforming triangulations.
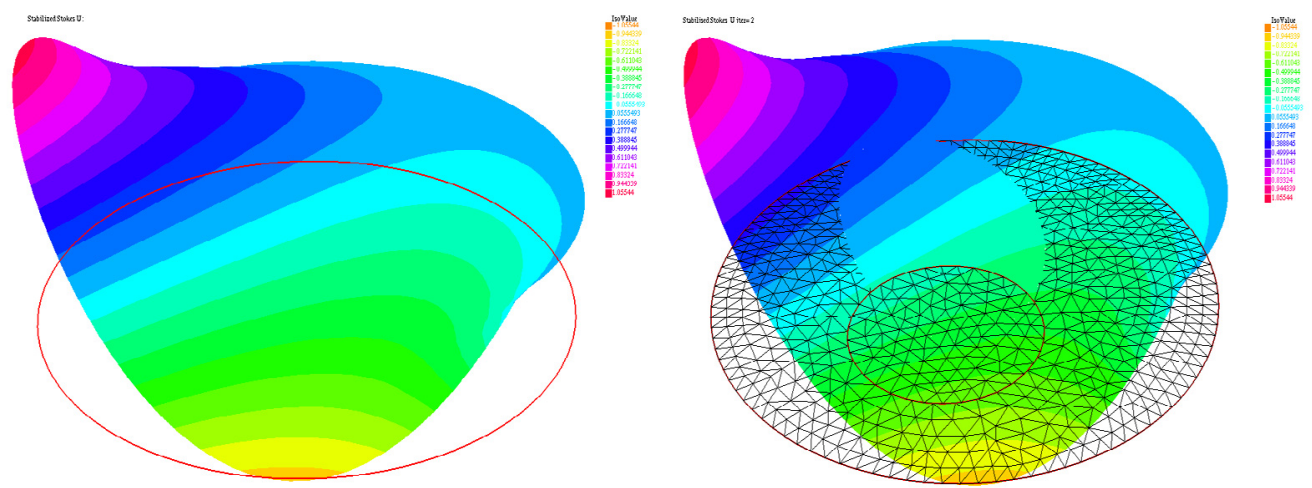

FiguRE 9. First component of velocity field for second test with non conforming triangulations.
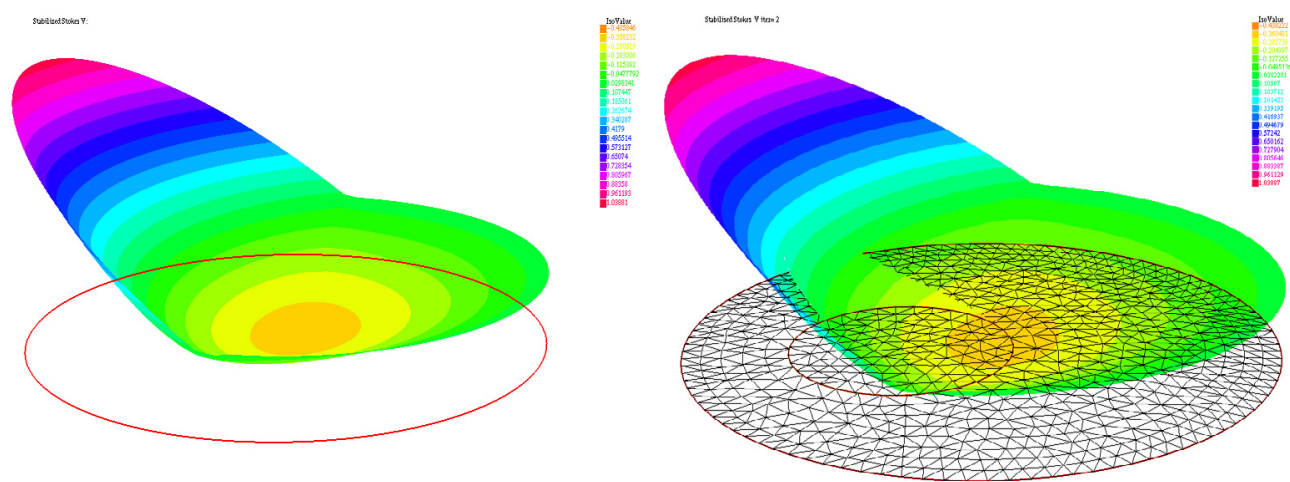

FiguRE 10. Second component of velocity field for second test with non conforming triangulations. 

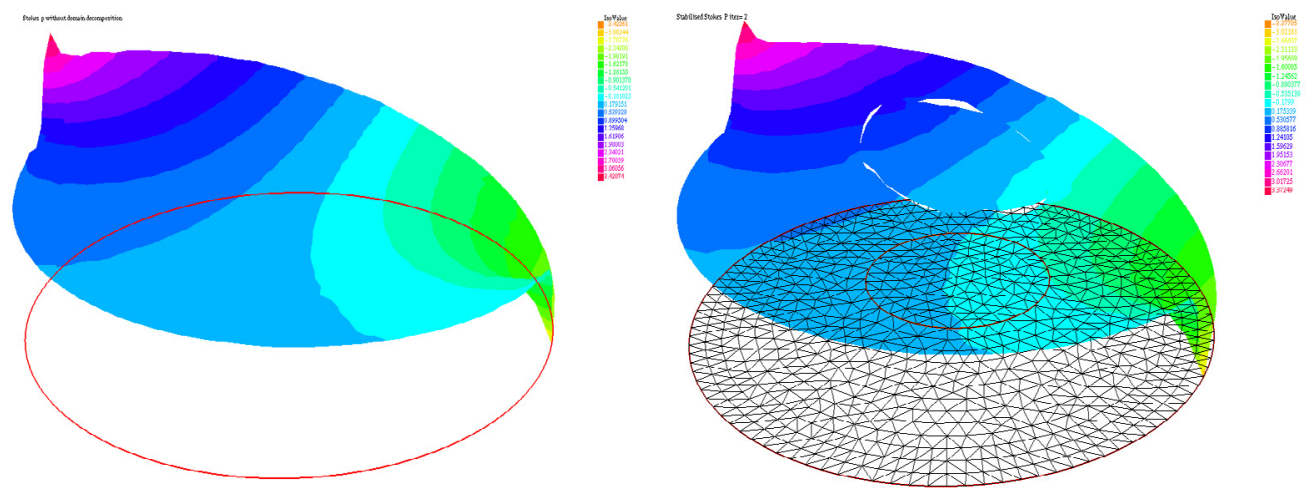

Figure 11. Computed pressure for second test with non conforming triangulations.

operators like Laplace [4,6] and incompressible Stokes [7]. The inclusion now of stabilization techniques for Stokes equations makes it also affordable for large problems.

Acknowledgements. The authors acknowledge the finantial support from Ministerio de Ciencia e Innovación of Spain via Research Project MTM2009-07719 and from Junta de Andalucia, Spain, via Research Project P07-FQM-02538.

\section{REFERENCES}

[1] R.A. Adams, Sobolev Spaces. In vol. 65 of Pure and Applied Mathematics. Academic Press, New York, London (1975).

[2] D. Braess, W. Dahmen and C. Wieners, A multigrid algorithm for the mortar finite element method. SIAM J. Numer. Anal. 37 (1999) 48-69.

[3] F. Ben Belgacem, The Mortar finite element method with Lagrange multipliers. Numerische Mathematik 84 (1999) $173-197$.

[4] C. Bernardi, T. Chacón Rebollo and E. Chacón Vera, A FETI method with a mesh independent condition number for the iteration matrix. Comput. Methods Appl. Mech. Engrg. 197 (2008) 1410-1429.

[5] C. Bernardi, Y. Maday and A.T. Patera, A new nonconforming approach to domain decomposition: the mortar element method, edited by H. Brezis and J.-L. Lions. Collège de France Seminar XI, Pitman (1994) 13-51.

[6] E. Chacón Vera, A continuous framework for FETI-DP with a mesh independent condition number for the dual problem. Comput. Methods Appl. Mech. Engrg. 198 (2009) 2470-2483.

[7] E. Chacón Vera and D. Franco Coronil, A non standard FETI-DP mortar method for Stokes Problem. Proceedings of the 3rd FreeFem++ days, Paris, 2011. J. Numer. Math. 20 (2012) 161-182.

[8] http://www.freefem.org/ff++

[9] L.P. Franca, T.J.R. Hughes and R. Stenberg, Stabilized Finite Element Methods, in Incompressible Computational Fluid Dynamics, chap. 4, edited by M. Gunzburger and R.A. Nicolaides. Cambridge Univ. Press, Cambridge (1993) 87-107.

[10] V. Girault and P.-A. Raviart, Finite Element Methods for Navier-Stokes Equations. Theory and Algorithms, vol. 5 of Springer Series in Comput. Math. Springer-Verlag, Berlin (1986).

[11] P. Grisvard, Singularities in Boundary value problems, vol. 22 of Recherches en Mathématiques Appliquées, Masson (1992).

[12] C.O. Lee and E.H. Park, A dual iterative substructuring method with a penalty term, Numerische Mathematik V. 112 (2009) 89-113.

[13] P.A. Raviart and J.-M. Thomas, Primal Hybrid Finite Element Methods for second order elliptic equations. Math. Comput. 31 (1977) 391-413. 